\title{
Post-infarctual left ventricular apical thrombosis successfully treated with low-dose apixaban: A case report
}

\author{
Antonio Gianluca Robles ${ }^{1 *}$; Paolo Pollice ${ }^{1 ; 2^{*}}$; Luca Amato ${ }^{1}$; Domenico Zanna ${ }^{1}$; Luigi Truncellito ${ }^{2}$ and Stefano
}

Favale $^{1}$.

${ }^{1}$ Institute of Cardiovascular Disease, Department of Emergency and Organs Transplantations, University of Bari "Aldo Moro", 70121 Bari, Italy.

${ }^{2}$ Unit of Cardiology, Civil Hospital “Giovanni Paolo II”, 75025 Policoro (MT), Italy

Address for correspondence:

1. Dr. Antonio Gianluca Robles, M.D., Institute of Cardiovascular Disease, Department of Emergency and Organs Transplantations, University of Bari "Aldo Moro", Piazza Giulio Cesare 11, CAP 70122, Bari, Italy, Tel: +39 3278678658; fax: +39 080/5478796; e-mail: gianlucarobles24@gmail.com; ORCID ID: 0000-0002-2150-3788

2. Dr. Paolo Pollice, Institute of Cardiovascular Disease, Department of Emergency and Organs Transplantations, University of Bari “Aldo Moro", 70121 Bari, Italy

Drs. Robles and Pollice gave the same contribute as first authors

Submitted: 21 April 2020

Approved: 30 April 2020

Published: 2 May 2020

How to cite this article: Robles AG, Pollice P, Amato L; Zanna D,Truncellito L, et. al. Post-infarctual left ventricular apical thrombosis successfully treated with low-dose apixaban: A case report. G Med Sci. 2020; 1(1): 001-003.

https://www.doi.org/10.46766/thegms.cardio.20042201

Copyright: @ 2020 Robles AG; Pollice P, et al. This is an open access article distributed under the Creative Commons Attribution License, which permits unrestricted use, distribution, and reproduction in any medium, provided the original work is properly cited.

\section{ABSTRACT}

The recommended treatment for ventricular thrombosis is based on vitamin $\mathrm{K}$ antagonists use for at least 3 months. Till now there are not completed trials that allow use of direct oral anticoagulants for this specific complication in neither chronic nor acute settings. We report the case of a subacute left ventricular apical thrombosis complicating a myocardial infarction in a 78-years-old patient effectively treated with low-dose apixaban (2.5 mg twice daily) associated with dual antithrombotic therapy. This off-label therapeutic approach was successful and no hemorrhagic complications neither thrombotic recurrences were noticed in the acute phase and at 2-years follow up. This case is the first one describing a successful and not complicated management of post-myocardial infarction ventricular thrombosis using off-label low-dose apixaban and it encourages further evidences and studies to evaluate the possibility of using this approach in the management of this kind of issues.

Keywords: apixaban; direct oral anticoagulants; triple antithrombotic therapy; left ventricular thrombosis; myocardial infarction complications.

\section{INTRODUCTION}

Ventricular thrombosis is a high-risk embolic condition and is potentially fatal. The recommended treatment is based on the use of vitamin $\mathrm{K}$ antagonists (VKAs), whose use is burdened by numerous adverse effects. An alternative to these may be the use of direct oral anticoagulants (DOACs) but till now there are not completed trials allowing their application for this specific complication. However, in literature there are several case reports showing the possibility of effectively treating ventricular thrombosis with DOACs. Our case is an example of effective and safe treatment of ventricular thrombosis with low dose apixaban and it gives further evidence and solidity to this therapeutic option. 


\section{CASE REPORT}

We present the case of a hypertensive and hyperlipidemic 78-years-old male patient with no previous cardiac diseases who presented to our Hospital with chest pain radiated to the left arm. He was admitted to Chest Pain Unit for anterolateral acute myocardial infarction with STsegment elevation (STEMI) and he was submitted to primary percutaneous coronary intervention and drug-eluting stent implantation in the proximal left anterior descending coronary artery. Dual antiplatelet therapy (DAPT) based on acetylsalicylic acid (ASA) $100 \mathrm{mg} /$ die and clopidogrel $75 \mathrm{mg} /$ die was soon established once a loading dose of ASA $250 \mathrm{mg}$ intravenous and clopidogrel $600 \mathrm{mg}$ per os had been administered. At the admission patient's weight was $75 \mathrm{Kg}$ and he had the following renal function parameters: serum creatinine $1.1 \mathrm{mg} / \mathrm{dl}$ and a CKD-EPI-estimated glomerular filtration rate of $63 \mathrm{ml} / \mathrm{min}$. The transthoracic echocardiogram (TTE), performed one day after revascularisation, showed akinesia of the whole apex and of the mid portions of the interventricular septum, anterior and antero-lateral wall and a $10 \times 8$ $\mathrm{mm}$ sized, ovoidal, hyperechoic, sessile formation into apex suggestive of thrombus (Figure 1). The patient, a Medical Doctor, refused administration of either VKAs or parenteral anticoagulation and left informed consent to an anticoagulation therapy with DOAC. Low dose regimen apixaban (2.5 mg twice daily) was prescribed taking into account the greater general augmented bleeding risk related to the triple antithrombotic therapy and a presumptive increased one due to a large pseudoaneurysm of the right radial artery complicating the cardiac catheterization. So the patient was discharged with the following therapy: DAPT for at least one year (ASA $100 \mathrm{mg}$ daily and clopidogrel $75 \mathrm{mg}$ daily) and apixaban $2.5 \mathrm{mg}$ twice daily. His serum creatinine at discharge was $1.4 \mathrm{mg} / \mathrm{dl}$. Apixaban therapy was stopped 30 days after the beginning of treatment when the disappearance of the hyperechoic image related to the thrombus and an improvement in ventricular contractility (apex became hypokinetic from akinetic) were documented at TTE (Figure 2). Moreover, pseudoaneurysm resolution was detected few days after discontinuation of apixaban. TTE confirmed the absence of the apical thrombus at 6-months and 2-years follow up. There were neither bleeding nor embolic complications during treatment and no hemorrhagic and thromboembolic complications were observed during the 2-years follow-up. The patient gave informed consent to the anonymous use of his clinical data for scientific purposes.

\section{DISCUSSION}

Left ventricular (LV) thrombus is a complication of acute myocardial infarction (MI) and it occurs in about $15 \%$ of patients with STEMI and up to $25 \%$ of patients with anterior MI [1]. Hypercoagulability, endothelial injury, and blood stasis, known as Virchow triad, play an important role in LV thrombus formation. LV dyskinesia or akinesia due to tissue necrosis lead to intracavitary blood stasis. The lesion of the subendothelial tissue causes inflammation and exposure to collagen, acting as a nest for platelets aggregation and activation of the coagulation cascade. The resulting LV thrombus consists of fibrin, platelets and red blood cells [1]. It requires prompt and adequate anticoagulation because it is associated with systemic thromboembolism risk. The recommended treatment for ventricular thrombosis is based on VKAs use for at least 3 months [2] while, till now, there are not in literature completed trials that allow the use of DOACs for this specific complication. However, cases of ventricular thrombosis successfully treated with dabigatran, rivaroxaban and apixaban were described, and in one case edoxaban was also used for this purpose. [3;4]. We can find different indisputable advantages encouraging the use of DOACs for the off-label treatment of ventricular thrombosis: fleeting INR, desire to avoid frequent blood tests, hemorrhagic problems, liver side effects, food restrictions, rejection or request by the patient [3]. The median duration of treatment with DOACs to treat ventricular thrombosis till complete resolution that we find in literature is 30 days [3], but in some cases it has been reported even in 1 week [5]. The reported case dates in October 2016 and, to the best of our knowledge, it represents the first one in which apixaban $2.5 \mathrm{mg}$ twice daily is used for off-label treatment of ventricular thrombosis. We choose this drug because it is characterized by good safety profile in terms of both major bleeding complications and gastrointestinal ones [6] and optimal patient's compliance [7]. Low-dose regimen was chosen taking into account the greater general hemorrhagic risk related to the triple antithrombotic therapy and, in particular, the specific alleged increased risk due to the possible rupture of the pseudoaneurysm. The efficacy of DOACs in the treatment of ventricular thrombosis does not appear to be influenced by concomitant single or dual anti-platelet therapy [8] and in our case we did not observe hemorrhagic complications. Apixaban effectiveness may be enhanced by its ability to direct inhibit activated factor $\mathrm{X}$ present into thrombus mass [6].

\section{CONCLUSIONS}

In summary, this case shows a successful and not complicated management of post STEMI myocardial infarction ventricular thrombosis using a triple therapy inclusive of off-label low-dose apixaban in a patient without DOAC dose-reduction recommended criteria. More and larger studies and clinical trials are required to demonstrate the efficacy and safety of DOACs in the specific above-mentioned clinical scenario.

\section{CONFLICT OF INTEREST}

The authors declare no competing interests.

\section{ACKNOWLEDGEMENTS}

None.

\section{REFERENCES}

1. McCarthy CP, Vaduganathan M, McCarthy KJ, Januzzi JL Jr, Bhatt DL, McEvoy JW. Left Ventricular Thrombus After Acute Myocardial Infarction: Screening, Prevention, and Treatment. JAMA Cardiol. 2018 Jul 1;3(7):642-649.

2. Guyatt GH, Akl EA, Crowther M, Guttermann DD, Schuünemann HJ. Executive summary: antithrombotic therapy and prevention of thrombosis, 9th ed: American College of Chest Physicians evidence-based clinical practice guidelines. Chest 2012 141:7S-47S.

3. Leow AS, Sia CH, Tan BY, Loh JP. A meta-summary of case reports of non-vitamin $\mathrm{K}$ antagonist oral anticoagulant use in patients with left ventricular thrombus. J Thromb Thrombolysis 2018 46:68-73.

4. Pei-Heng Kao, Ping-Yin Chou, Po-Chao Hsu, Tien-Chi Huang. Resolution of left ventricular thrombus by edoxaban after failed treatment with warfarin overdose: A case report. Medicine. 2019 Jan 98(2):e14065.

5. Nakasuka K, Ito S, Noda T, Hasuo T, Sekimoto S, Ohmori H et al. Resolution of left ventricular thrombus secondary to tachycardiainduced heart failure with rivaroxaban. Case Rep Med 2014 Article ID 814524

6. Ferri N, Corsini A. Nuovi anticoagulanti orali: considerazioni di farmacologia clinica. G Ital Cardiol 2015 16:3S-16S.

7. Lip GYH, Pan X, Kamble S, Kawabata H, Mardekian J, Masseria C et al. Discontinuation risk comparison among 'real-world' newly anticoagulated atrial fibrillation patients: Apixaban, warfarin, dabigatran, or rivaroxaban. PLoS One 2018 13(4):e0195950.

8. Maniwa N, Fujino M, Nakai M, Nishimura K, Miyamoto Y, Kataoka $Y$ et al. Anticoagulation combined with antiplatelet therapy in patients with left ventricular thrombus after first acute myocardial infarction. Eur Heart J 2018 39:201-8. 


\section{FIGURES}

\section{FIGURE 1. Ventricular apical thrombosis.}

Apical three-chamber view of the left ventricle showing a 10 × $8 \mathrm{~mm}$ sized, ovoidal, hyperechoic, sessile mass (indicated by arrows) referable to apical thrombus on the second day after PCI for acute anterior myocardial infarction; the ventricular apex was akinetic.

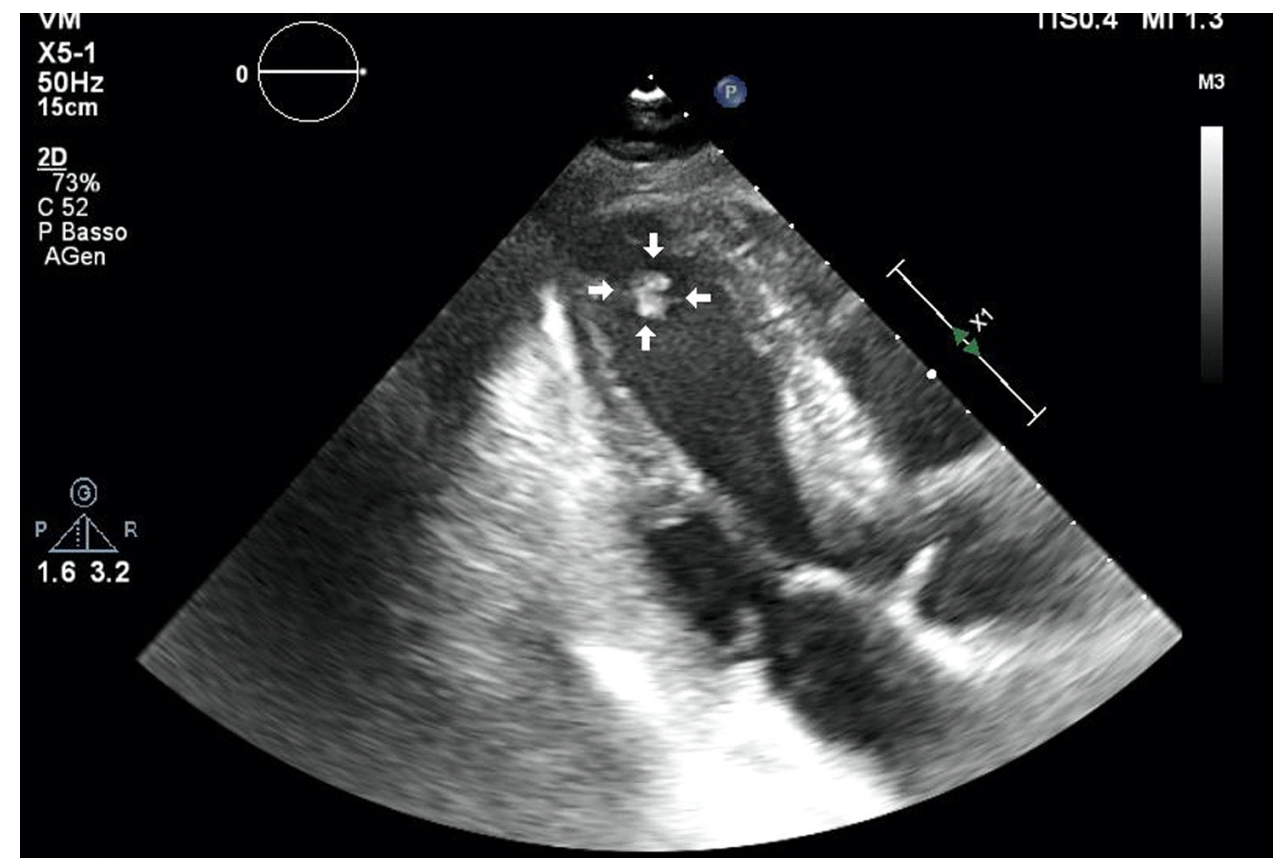

Fig 1

FIGURE 2. Ventricular apical thrombosis resolution.

The same apical three-chamber echocardiographic view seen in Fig. 1 now shows ventricular thrombosis resolution after treatment with apixaban $2.5 \mathrm{mg}$ twice daily for 30 days.

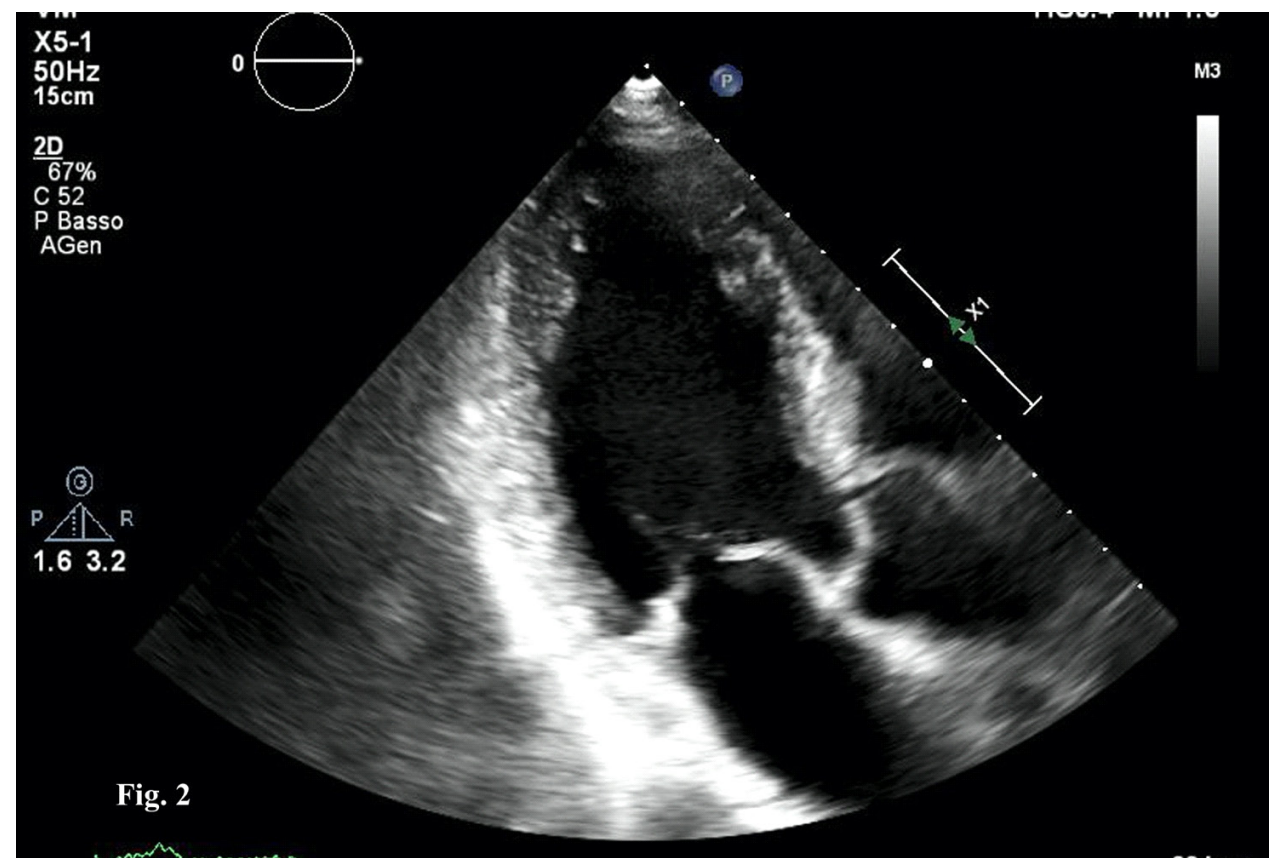

Fig 2 